\title{
Health-related quality of life among osteoarthritis patients attending primary care clinics of Mangalore city
}

H. N. Harsha Kumar', K. Nagaraj, Kritika Luthra', Parul Gupta', Poornima Sapar', Suvigya Gupta ${ }^{1}$, Anuj Tyagi ${ }^{1}$

Departments of Community Medicine, Kameneni Institute of Medical Sciences, Narketpally,

Nalgonda, Andhra Pradesh,

${ }^{1}$ Kasturba Medical College [Manipal University], Mangalore, Karnataka, India

Address for the Correspondence:

Dr. H. N. Harsha Kumar,

Department of Community Medicine, Kasturba Medical College, Near Hampanakatta, Mangalore - 575 001, Karnataka,

India.

E-mail: hnkswamy@gmail.com

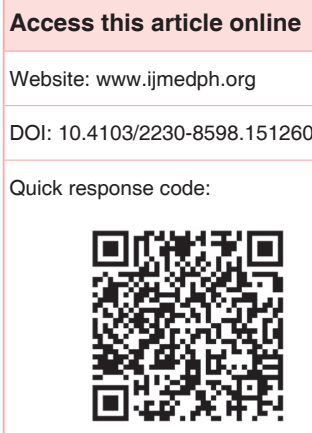

Introduction: Osteoarthritis (OA) of the knee is the most common type of arthritis of the elderly among Asians. Reports suggest that it affects the quality of life. Hence, this study was undertaken with the objective to know the impact of OA of the knee on 8 domains of health related quality of life (HROoL) which include: (1) Physical functioning (PF), (2) role limitation due to physical health (RP). (3) Emotional well-being/mental health $(\mathrm{MH})$, (4) role limitation due to emotional problems (RE), (5) energy/vitality, (6) social well-being (short form [SF]), (7) body pain, (8) general health. Materials and Methods: This cross-sectional study was conducted in primary care rural clinics situated on peripheral areas of Mangalore city. Total sample size was calculated to be 77. Patients who met the criteria of modified American Rheumatologic Association Classification for OA of knee were administered a SF36 questionnaire to measure the $\mathrm{HROoL}$ in the local language version. Mean scores (with its standard deviation) across each domain was calculated. Results: Of 80 patients, 38 were males. OA affected HRQoL. Four out 8 factors had more impact on HRQoL (they are PF, RP, MH, RE). Duration of OA is an important determinant on HRQoL. Patients with newer onset of OA ( $<4$ years) had less physical impact on (Domain, Mean) (PF, 54.36; RP, 13.5) and more emotional/mental impact $(\mathrm{MH}$, 28.23; RE, 59.13). With increasing duration ( $>4$ years) of OA (Domain, Mean), the emotional impact $(\mathrm{MH}, 44.67 ; \mathrm{RE}, 45.67)$ reduced but the physical impact (PF, 47.83; RP, 19.75) persisted/got worse. Conclusion: Osteoarthritis had impact on HRQoL. Duration of OA determines the kind of impact. With the onset of OA emotional/mental support would be needed in addition to the medical treatment. As the disease progresses, they tend to cope with emotional/mental problems even as the physical problems persist/increase.

Key words: Geriatrics, health related quality of life, osteoarthritis, primary care

\section{INTRODUCTION}

Osteoarthritis $(\mathrm{OA})$ is the most common type of arthritis found worldwide especially in the elderly. ${ }^{[1]}$ With the population ageing, the prevalence of $\mathrm{OA}$ is increasing, and its consequences have a significant impact on the society. ${ }^{[1]} \mathrm{It}$ is a disease characterized by pain and inflammation due to the involvement of the articular cartilage, soft tissues and bone. ${ }^{[2]}$ Older people with this disease also experience depression, diminished quality of life and economic hardship. ${ }^{[3]}$

Of all the types, OA of the knee is more common in Asian region. ${ }^{[4]}$ There are two published evidences to suggest that OA (involving any of joints) is known to impact the quality of life. ${ }^{[5,6]}$ But these were conducted in the tertiary/secondary care settings. But when we take OA of Knee into consideration, we find that there are only two studies that have specifically examined the impact on quality of life among elderly in primary care setting. A study on $\mathrm{OA}$ in primary care setting from America has reported significant impact on various aspects of quality of life like physical functioning (PF), emotional behavior, sleep, etc. ${ }^{[7]}$ Another similar study done in primary care setting in Malaysia has reported significant impact of $\mathrm{OA}$ on quality of life. ${ }^{[8]} \mathrm{OA}$ was also reported to affect the psychological functioning and associated with depression among Chinese patients reporting to primary care setting. ${ }^{\left[{ }^{[9]}\right.}$ This study from china examined the overall impact of many chronic diseases in addition to OA. So it's important to 
assess the quality of life among OA patients. This helps in identifying treatment and counseling needs of these patients.

There are no reported studies on the impact of OA of the knee in primary care setting from India. The study was conducted with the objective of determining the impact of OA of the knee on healthrelated quality of life (HRQoL) among patients attending primary care clinics in the outskirts of Mangalore city. The objectives were to know the impact of OA on 8 domains of HRQoL.

\section{MATERIALS AND METHODS}

\section{Study design}

A questionnaire based cross-sectional study.

\section{Study setting}

Department of Community Medicine, Kasturba Medical College, has established primary care outreach clinics in the peripheral areas of Mangalore city. These clinics are located at Boloor, Asaigoli, Shivnagar, Jeppinamogaru and Lady Hill areas. These clinics provide outpatient services.

\section{Study subjects}

Patients with $\mathrm{OA}$ attending the outreach clinics in the month of February 2011 were chosen as our study subjects.

\section{Inclusion criteria}

All patients who meet the criteria of modified American Rheumatologic Association Classification for OA of the knee were included in the study. This would include patients with knee pain for about 1 -month or more, aged $>50$ years.

\section{Exclusion criteria}

Patients whose knee pain was because of conditions other than OA were excluded.

\section{Sample size}

Sample size was calculated using the formula for infinite population $n=4\left(\sigma^{2} / \mathrm{E}^{2}\right)$. For mean duration of pain of 4.07 years (standard deviation [SD] 2.96), the relative precision of $20 \%$, at $95 \%$ confidence interval, a sample size of 54 was arrived at. ${ }^{[8]}$ Accounting for $30 \%$ nonresponse the total sample size came to be 77 .

\section{Sampling}

Nonrandom sampling. The patients who met the study criteria were sequentially included.

\section{Study instrument}

The medical outcome study 36-item short form (social well-being $[\mathrm{SF}]-36)$ questionnaire was used to measure the HRQoL in this study ${ }^{[10]}$ It is a reliable and validated generic instrument that has been used extensively to measure HRQoL in diverse groups. This
36 item questionnaire consists of questions related to 8 domains. They are:

1. PF,

2. role limitation due to physical health (RP).

3. Emotional well-being/mental health $(\mathrm{MH})$,

4. role limitation due to emotional problems (RE),

5. energy/vitality (VT),

6. social well-being (SF),

7. body pain,

8. general health $(\mathrm{GH})$.

Most of these domains of quality of life are scored from 0 to 100 (Except MH 16-92; VT 10-90; GH 6-80).

Information about sociodemographic characteristics was also collected.

This questionnaire was translated by a linguistic expert to Kannada version (local language) and back-translated to English version by another expert who was not acquainted with original English version. The original and the back-translated versions were compared for conceptual equivalence.

\section{Pilot testing}

This was a pilot tested in our clinics. It was found to be suitable for use.

\section{Data collection}

After obtaining permission from institutional ethics committee, the patients were approached in the outreach clinics. Informed consent was taken before administering the questionnaire. Confidentiality of responses was assured as the questionnaire was anonymous. About 40 min time was allotted for them to fill the questionnaire. Clarification if any sought by the patients were attended to by the Medico-social Workers. Junior resident doctors ascertained the severity of the disease.

\section{Data analysis}

Data were entered in SPSS 12.0 version. Results are expressed as proportions in appropriate tables. Mean scores and its SD for all the domains of HRQoL were calculated. Student's independent $t$-test was used to compare the means. $P<0.05$ was considered as statistically significant.

\section{RESULTS}

Totally 80 patients attending a primary care clinics met the study criteria. The proportion of females $(n=42,52.5 \%)$ was higher than males $(n=38,47.5 \%)$. Hindus constituted the majority $(n=64,80 \%)$, followed by Christians $(n=9,11.3 \%)$ and Muslims $(n=7,8.8 \%)$. Majority (85\%) were married. About half of them (51.3\%) were living with relatives as compared to those living alone (16.2\%). Old age homes supported the lives of $32.5 \%$. Most of them had primary school education. About a third (35\%) of study subjects did not go to school. 
Nearly half of them $(n=41,51.25 \%)$ had pain for $>4$ years. About $37.5 \%$ of them had one or more co-morbid condition. The distribution of co-morbid conditions was as follows (\%): Cataract (25), hypertension (20.1), diabetes (15), and coronary heart disease (15).

The scores obtained for various aspects of quality of life presented in Table 1 indicate that the PF is good, and role limitation due to physical condition is lower. Comparison of patients with lesser duration of disease ( $\leq 4$ years) with those of higher duration ( $>4$ years) presented in Table 2 shows that 4 out 8 domains of HRQoL were significantly affected. They are (1) PF, (2) RP. (3) Emotional well-being MH, (4) RE.

No significant gender differences were observed across various domains of HRQoL. As the majority of the patients did not have Co-Morbid conditions, no comparisons were made between those who had and did not have co-morbidity. For similar reasons comparison was not done between those living alone and those with social support.

\section{DISCUSSIONS}

Results indicate that OA had impact on HRQoL. It was also seen that the impact was mainly influenced by duration of disease. Hence, the discussion below is focused on this aspect with respect of HRQoL.

\section{Duration of osteoarthritis on physical functioning}

As the duration of disease increased, the PF decreased, and the RP increased. de Bock et al. have reported that older patients had a higher impact on PF when compared to younger patients. ${ }^{[7]}$ Zakaria et al. have reported a significant negative correlation between age and PF. However, they have not provided any analysis with respect to duration of disease. ${ }^{[8]}$ As OA progresses, disabilities are likely to develop, so a decline in PF is expected.

\section{Duration of osteoarthritis on emotional well-being/ mental health}

Patients with lesser duration have lower emotional well-being and higher limitation due to emotional problems. There are no comparable studies to explain the pattern of impact on emotional aspects of quality of life. It can be inferred that with increasing duration of disease the people have mentally adjusted themselves so they scored higher for emotional well-being and perceived lesser RE. Whereas the people with relatively new onset of OA could not mentally cope with it and scored lower on emotional well-being.

As the majority of the patients did not have any co-morbidity, the impact on HRQoL could be attributed mainly due to OA.

\section{Social support}

Few $(16.2 \%)$ patients lived alone. Most of them had support from family/relatives or old age home. Though we have not compared between these two categories, it can be seen that, scores obtained are not higher than other components. One would have expected that social support would improve the care and bring a sense of security in old age. But, the results do not reflect that. The reason is not clear.

\section{Limitations}

We cannot rule out recall bias particularly among individuals aged $>65$ years. This is not a community-based study. But our primary care clinics were situated in rural areas and provided care to these cases with OA. So, the impact of bias could be minimal.

\begin{tabular}{lcccc} 
Table 1: Scores obtained under various aspects of quality of life & & \\
\hline Factors determining HRQoL & Minimum score & Maximum score & Mean & Standard deviation \\
\hline Physical functioning & 0 & 95 & 51.18 & 6.91 \\
Role limitation due to physical health & 0 & 100 & 16.62 & 3.09 \\
Emotional well-being/mental health & 16 & 92 & 36.45 & 5.92 \\
Role limitation due to emotional problems & 0 & 100 & 52.45 & 4.95 \\
Energy/vitality & 10 & 90 & 49.40 & 18.64 \\
Social well-being & 0 & 100 & 40.25 & 26.05 \\
Bodily pain & 0 & 100 & 50.93 & 26.24 \\
General health & 6 & 80 & 49.38 & 17.51 \\
\hline
\end{tabular}

HRQoL = Health related quality of life

\begin{tabular}{lccc} 
Table 2: Comparison of mean scores of HRQoL according to duration of disease \\
\hline Factors determining HRQoL & $\mathbf{\leq 4}$ years $(\boldsymbol{n}=\mathbf{3 9})$ & $\mathbf{> 4}$ years $(\boldsymbol{n}=\mathbf{4 1})$ & Student's independent t-test $(\boldsymbol{P})$ \\
\hline Physical functioning & 54.36 & 47.83 & $2.47(0.016)$ \\
Role limitation due to physical health & 13.5 & 19.75 & $6.52(0.0001)$ \\
Emotional well-being/mental health & 28.23 & 44.67 & $9.93(0.0001)$ \\
Role limitation due to emotional problems & 59.13 & 45.67 & $8.13(0.0001)$ \\
Energy/vitality & 48.2 & 50.8 & $1.57(0.12)$ \\
Social well-being & 41.25 & 39.25 & $1.2(0.23)$ \\
Bodily pain & 51.95 & 49.91 & $1.23(0.22)$ \\
General health & 48.32 & 50.44 & $1.28(0.2)$
\end{tabular}

HRQoL = Health related quality of life 


\section{CONCLUSION}

Osteoarthritis had impact on HRQoL. Duration of disease played a role in HRQoL. Patients with relatively recent onset of disease had less physical impact and more emotional impact. As the disease progresses, they cope mentally though the physical limitation increases and PF decreases. OA patients need emotional/mental and social support in addition to the medical treatment.

\section{ACKNOWLEDGMENT}

We wish to thank our study subjects who devoted their precious time for our study and the medico-social workers of the Department of Community Medicine, KMC Mangalore who helped us in administering and explaining the questionnaire to our study subjects.

\section{REFERENCES}

1. Brooks PM. Impact of osteoarthritis on individuals and society: How much disability? Social consequences and health economic implications. Curr Opin Rheumatol 2002;14:573-7.

2. De Filippis L, Gulli S, Caliri A, Romano C, Munaò F, Trimarchi G, et al. Epidemiology and risk factors in osteoarthritis: Literature review data from "OASIS" study. Reumatismo 2004;56:169-84.

3. Blixen CE, Kippes C. Depression, social support, and quality of life in older adults with osteoarthritis. Image J Nurs Sch 1999;31:221-6.
4. Veerapan K. Osteoarthritis-Asian perspective. In: Howe HS, Feng PH, editors. Textbook of Clinical Rheumatology. Singapore: National Arthritis Foundation; 1997. p. 294-5.

5. Jadhav MP, Jadhav PM, Mutke AP, Sonawane SD, Patil BD, Naik NB, et al. A prospective observational study to assess quality of life and prescription pattern in osteoarthritis patients at tertiary health centre in Mumbai. Indian J Med Sci 2011;65:58-63.

6. Woo J, Lau E, Lee P, Kwok T, Lau WC, Chan C, et al. Impact of osteoarthritis on quality of life in a Hong Kong Chinese population. J Rheumatol 2004;31:2433-8.

7. de Bock GH, Kaptein AA, Touw-Otten F, Mulder JD. Health-related quality of life in patients with osteoarthritis in a family practice setting. Arthritis Care Res 1995;8:88-93.

8. Zakaria ZF, Bakar AA, Hasmoni HM, Rani FA, Kadir SA. Health-related quality of life in patients with knee osteoarthritis attending two primary care clinics in Malaysia: A cross-sectional study. Asia Pac Fam Med 2009;8:10.

9. Lam CL, Lauder IJ. The impact of chronic diseases on the health-related quality of life $(\mathrm{HRQOL})$ of Chinese patients in primary care. Fam Pract 2000;17:159-66.

10. Medical Outcomes Trust. Literature construction of SF 36 version 2-Psychometric considerations, translations and discussions. Available from: http://www.sf-36.org/tools/SF36.shtml/. [Last accessed on 2012 Feb 02].

How to cite this article: Harsha Kumar HN, Nagaraj K, Luthra K, Gupta P, Sapar P, Gupta S, et al. Health-related quality of life among osteoarthritis patients attending primary care clinics of Mangalore city. Int J Med Public Health 2015;5:55-8.

Source of Support: Nil, Conflict of Interest: None declared. 